\title{
Derivation and Application of the Fokker-Planck Equation to Discrete Nonlinear Dynamic Systems Subjected to White Random Excitation
}

\author{
'Thomas K. CaUghey \\ California Instimte of Technology, Pasudenu, Californic
}

\begin{abstract}
The Fokker-Planck equation is dcrived and applied to discrete nonlinear dynamic systems subjected to white random excitation. For the class of problems in which the nonlincarities involve only the displacements of the system, it is shown that exact solutions can be constructed for the stationary Fokker-Planck equation. It is further shown that if stationary solutions exist they are unique.
\end{abstract}

\section{INTRODUCTION}

$\mathbf{R}^{\mathrm{H}}$ ECENT developments in jet and rocket propulsion have given rise to new problems in mechanical and structural vibrations. The pressure fields generated by these devices fluctuate in a random manner and contain a wide spectrum of frequencies that may result in severe vibration in the aircraft or missile structure. As more data are gathered on strong-motion earthquakes, it is becoming apparent that earthquakes are examples of random processes that may excite severe vibration and even failure in buildings and other structures. Measurements of the motion of ships in a confused sea or aircraft flying through turbulent air reveal that such motions can be described only statistically.

The examples given above have two things in common: (a) they involve the response of mechanical systems to random excitation; (b) in general, they involve nonlinear behavior, since almost all real physical systems exhibit nonlinearity for suficiently large motions.

The theory of linear systems subjected to random excitation is well-developed, ${ }^{1-4}$ and, though there still remain many unanswered questions, we can answer

\footnotetext{
1 J. S. Benclat, Principles and A pplicalions of Random .Vise Theory (John Wiley \& Sons, Inc., New York, 1955).

${ }^{2}$ J. H. Laning and R. H. Battin, Random Processes in A ulomalic Control (Mcciraw-Hill Book Co., Inc., New York, 1956).
}

most of the questions of practical interest. In the case of nonlinear systems, however, the standard techniques of linear analysis cannot be applied, though approximate methods have been developed to extend linear analysis to certain systems containing small nonlinearities. ${ }^{5}$ ? The purpose of this paper is to deal with a different approach to the analysis of linear and nonlinear systems, based on the theory of Markoff random processes. We show that the behavior of discrete dynamic systems subjected to white random excitation are examples of continuous multidimensional Markoff processes. Such processes are completely characterized by their transitional or conditional probability law, which is obtained as the fundamental solution to the Fokker-Planck equation appropriate to the dynamic system. It is shown that exact stationary solutions may be constructed for a class of nonlinear problems in which the nonlinearity is it function only of the displacements. Furthermore, it is shown that if stationary solutions exist they are unique.

\footnotetext{
${ }^{3}$ W. B. Davenport and W. L. Root, Random Signals and Yoise (McGraw-Hill Book Co., Inc., New York. 1958).

'S. H. Crandall el al., Random Vibralions (Technology Press, (ambridge, Mass., and John Wiley \& Sons, Inc., New York, 1958).

sR. C. Buoton, "Noniinear Control Sustems with Random Injuts," IRE 'Trans. Circuit Theory 1, 9-18 (1954).

${ }_{6}^{6} T$. K. Caughey, "Response of a Nonlinear String to Random I,oarling," J. Appli. Mech. 26, 341-344 (1959).

${ }^{7 T} \mathrm{~T} . \mathrm{K}$. Caughey, "Random Excitation of a Isouded Nonlinear String," J. Appl. ilech. 27, 575-578 (1960).
} 


\section{FOKKER-PLANCK EQUATION}

\section{A. Basic Concepts of Probability Theory}

Roughly speaking, what is meant by a random excitation is one in which the forcing function does not depend in a completely definite way on the independent variable, time, as in a casual process. On the contrary, one gets in different observations different functions of time, so that only the probability is directly observable. The following set of probability distributions completely defines a random function ${ }^{8}$ :

$p_{1}(y t) d y=$ probability of finding $y$ in the range from $y$ to $y+d y$ at time $l$.

$p_{2}\left(y_{1} \iota_{1}, y_{2} \iota_{2}\right) d y_{1} d y_{2}=$ joint probability of finding $y$ in the range from $y_{1}$ to $y_{1}+d y_{1}$ at time $t_{1}$ and in the range from $y_{2}$ to $y_{2}+d y_{2}$ at time $\iota_{2}$.

$p_{3}\left(y_{1} \iota_{1}, y_{2} \iota_{2}, y_{3} \iota_{3}\right) d y_{1} d y_{2} d y_{3}=$ joint probability of finding $y$ in the range from $y_{1}$ to $y_{1}+d y_{1}$ at time $t_{1}$, in the range from $y_{2}$ to $y_{2}+d y_{2}$ at time $t_{2}$, and in the range from $y_{3}$ to $y_{3}+d y_{3}$ at time $l_{3}$.

The higher probability densities $p_{n}$, where $n=4,5$, $6, \cdots$, are defined in a similar manner. Each $p_{n}$ must satisfy the following conditions:

(a) $p_{n} \geqq 0$.

(b) $p_{n}$ is symmetric in $y_{1} t_{1}, y_{2} t_{2}, \cdots, y_{n} t_{n}$.

(c) $p_{k}=\int \cdots \int_{n-k} p_{n} d y_{k+1} \cdots d y_{n}$

Condition (c) is the important equation for determining a marginal probability.

The probability density $p_{n}$ can be used as a means of classifying a random function. The simplest case is that of a purely random function. This means that the value of $y$ at some time $t_{1}$ does not depend upon, or is not correlated with, the value of $y$ at any other time $t_{2}$. The probability distribution $p_{1}(y t) d y$ completely describes the function in this case, since the higher distributions are found from the following equation:

$$
p_{n}\left(y_{1} t_{1}, y_{2} t_{2}, \cdots, y_{N} t_{N}\right)=\prod_{i=1}^{N} p_{1}\left(y_{i} t_{i}\right) \text {. }
$$

The next more complicated case is where the probability density $p_{2}$ completely describes the functions. This is the so-called Markoff process. To define a Markoff process more precisely, we introduce the idea of the conditional probability. We define $p_{c 2}\left(y_{1} \mid y_{2}, t\right) d y_{2}$ as probability that, for a given $y=y_{1}$ at $t=0$, we find $y$ in the range from $y_{2}$ to $y_{2}+d y_{2}$ at a time $t$ later. We

"M. C. Wang and G. E. Uhlenbeck, "On the Theory of Brownian Motion II," Rev. Mod. Phys. 17, 323-342 (1945). [Also N. Wax et al., Selected Papers on Noise and Stochastic Processes (Tover Publications, Inc., New York, 1954), Pl. 113-132.] find $p_{c 2}$ by the relation

$$
p_{2}\left(y_{1} t_{1}, y_{2} t_{2}\right)=p_{1}\left(y_{1} t_{1}\right) p_{c 2}\left(y_{1} \mid y_{2}, t_{2}-t_{1}\right) .
$$

Equation (1.2) is the analogous to the joint probability of two dependent events:

$$
P(A B)=P(A) P(A \mid B),
$$

where $P(A B)$ is the probability of events $A$ and $B$ both occurring, $P(A)$ the probability of events $A$ occurring, and $P(A \mid B)$ the probability of event $B$ occurring on the given condition that event $A$ has already occurred.

Then, $P(A B)$ is the analog of $p_{2}, P(A)$ is the analog of $p_{1}$, and $P(A \mid B)$ is the analog of $p_{c 2}$.

The function $p_{c 2}$ must satisfy the conditions

(d) $p_{c 2}\left(y_{1} \mid y_{2} l\right) \geqq 0$

(e) $\int p_{c 2}\left(y_{1} \mid y_{2} t\right) d y_{2}=1$;

(f) $\quad p_{1}\left(y_{2} \iota_{2}\right)=\int p_{1}\left(y_{1} \iota_{1}\right) p_{c 2}\left(y_{1} \mid y_{2}, t_{2}-l_{1}\right) d y_{1}$

We can now define the Markoff process to mean that the conditional probability that $y$ lies in the interval, from $y_{1}$ to $y_{1}+d y_{1}$ at $t_{1}$, from $y_{2}$ to $y_{2}+d y_{2}$ at $t_{2}, \cdots$ from $y_{n-1}$ to $y_{n-1}+d y_{n-1}$ at $t_{n-1}$, depends only on the values of $y$ at $t_{n}$ and $t_{n-1}$. That is, for a Markoff process

$$
\begin{array}{r}
p_{c n}\left(y_{1} t_{1}, y_{2} t_{2}, \cdots, y_{n-1} t_{n-1} \mid y_{n} t_{n}\right) \\
=p_{c 2}\left(y_{n-1} t_{n-1} \mid y_{n} t_{n}\right) .
\end{array}
$$

It is now possible to derive $p_{3}, p_{4}, \cdots$ from $p_{2}$ and Eq. (1.2). For example:

$$
\begin{aligned}
& p_{3}\left(y_{1} t_{1}, y_{2} t_{2}, y_{3} t_{3}\right)=p_{2}\left(y_{1} t_{1}, y_{2} t_{2}\right) p_{c 2}\left(y_{2} t_{2} \mid y_{3} t_{3}\right) \\
&=\frac{p_{2}\left(y_{1} t_{1}, y_{2} t_{2}\right) p_{2}\left(y_{2} t_{2}, y_{3} t_{3}\right)}{p_{1}\left(y_{2} t_{2}\right)} \\
& p_{4}\left(y_{1} t_{1}, y_{2} t_{2}, y_{3} t_{3}, y_{4} t_{4}\right)=p_{3}\left(y_{1} t_{1}, y_{2} t_{2}, y_{3} t_{3}\right) p_{c 2}\left(y_{3} t_{3} \mid y_{4} t_{4}\right) \\
&=\frac{p_{2}\left(y_{1} t_{1}, y_{2} t_{2}\right) p_{2}\left(y_{2} t_{2}, y_{3} t_{3}\right)}{p_{1}\left(y_{2} t_{2}\right)} \\
& \times \frac{p_{2}\left(y_{3} l_{3}, y_{4} t_{4}\right)}{p_{1}\left(y_{3} t_{3}\right)}
\end{aligned}
$$

the latter from Eqs. (1.4) and (1.2).

In addition to conditions (d)-(f) on $p_{c 2}$, it must also satisfy the condition

$p_{c 2}\left(y_{1} \mid y_{2} l\right)=\int p_{c 2}\left(y_{1} \mid y \tau\right) p_{c 2}\left(y \mid y_{2}, t-\tau\right) d y$

$$
(0 \leqq \tau<t) .
$$

This equation has been alternately called the Smoluchowski equation and the Chapman-Kolmogorov 
equation. It implies that, when $y$ follows any path from $y_{1}$ at time zero to $y_{2}$ at time $t$ later, the particular path of $y$ at time $r$ is unimportant. Equation (1.6) is scen to integrate the probability over any path selected.

The next step would be to consider processes that are completely described by $p_{3}, p_{4}, \cdots$. Physically, there are few examples studied that involve these higher-order processes. Sometimes, when a process is not Markovian, we can find another variable $z$, which combined with $y$, makes a Markoff process. The variable $z$ may be $\dot{y}=d y / d t$ or another coordinate. In this case, the Smoluchowski equation becomes

$p_{c 2}\left(y_{1} z_{1} \mid y_{2} z_{2}, l\right)=\iint p_{c 2}\left(y_{1} z_{1} \mid y z, \ell_{1}\right)$

$$
X p_{c 2}\left(y z \mid y_{2} z_{2}, l-l_{1}\right) d y d z .
$$

Equation (1.i) may be generalized to problems involving.$T$ coordinates in place of the two used above. The general form of the equation is then

$$
\begin{aligned}
& p_{c 2}\left(\mathrm{y}_{1} \mid \mathrm{y}_{2}, l\right)=\int_{X-\text { fold }} \ldots \int_{i=1}^{N} \prod_{e 2}^{N} p_{1}\left(\mathrm{y}_{1} \mid \mathrm{z}, t_{1}\right) \\
& \times p_{c 2}\left(\mathbf{z} \mid \mathrm{y}_{2}, l-l_{1}\right) d z_{i},
\end{aligned}
$$

where $y$ is the position vector of a point in $X$-dimensional phase space and the integral is extended over all the phase space.

\section{B. Derivation of the Fokker-Planck Equation}

In order to derive the Fokker-Planck equation, the following assumptions must be made. The first and second incremental statistical moments of the displacement of the phase point in an infinitesimal period of time are

$$
\begin{aligned}
A_{i}(\mathrm{y}, t)=\int_{N-\text { folll }} \ldots \int\left(z_{1}-y_{1}\right) p_{c 2}(\mathrm{y} \mid \mathrm{z}, \Delta l) \prod_{i=1}^{N} d z_{i} ; \\
B_{i j}(\mathrm{y}, l)=\int_{N-\text { follt }}^{\ldots} \int\left(z_{i}-y_{i}\right)\left(z_{j}-y_{j}\right) \\
\quad \times p_{c 2}(\mathrm{y} \mid \mathrm{z}, \Delta l) \prod_{i=1}^{N^{v}} d z_{i} ;
\end{aligned}
$$

where $(i, j=1,2, \cdots, \lambda)$. The assumption is made that as $\Delta l \rightarrow 0$ only these moments of the displacement of the phase point become proportional to $\Delta l$, so that the following limits exist:

$$
\begin{aligned}
& a_{i}(\mathrm{y}, l)=\lim _{\Delta t \rightarrow 0} \frac{A_{i}}{\Delta \ell} \\
& b_{1 j}(\mathrm{y}, l)=\lim _{\Delta t \rightarrow 0} \frac{B_{i j}}{\Delta l} ;
\end{aligned}
$$

and that the higher moments are of the order of $(\Delta t)$. Physically, this implies that in a small time interval the coordinates of the phase point can change only by small amounts, and that is tantamount to the assumption of a Gaussian or normal probability distribution for the disturbances acting on the system.

Let us consider the Smoluchowski equation written in the form

$$
\begin{aligned}
p_{c}(\mathbf{x} \mid \mathbf{y}, t+\Delta l)=\int_{N-\text { lold }} \ldots \int & \prod_{i-1}^{N} d z_{i} \\
& \times p_{c}(\mathbf{x} \mid \mathbf{z}, l) p_{c}(\mathbf{z} \mid \mathbf{y}, \Delta l),
\end{aligned}
$$

where $\mathbf{z}$ at time $t$ is a point in phase space on any path of $\mathrm{y}$ from $\mathrm{x}$ at time zero to $\mathrm{y}$ at time $\iota+\Delta \ell$ later. Now, let $R(\mathrm{y})$ be an arbitrary scalar function of the variables $y_{1}, y_{2}, \cdots, y_{N}$, such that $R(\mathbf{y}) \rightarrow 0$ as all $y_{i} \rightarrow \pm \infty$. Multiplying Eq. (1.12) by $R(y)$ and integrating over the phase space,

$$
\begin{aligned}
& \int_{N \text {-rold }} \ldots \int R(\mathrm{y}) p_{c}(\mathrm{x} \mid \mathrm{y}, \imath+\Delta l) \prod_{i=1}^{N} d y_{i}
\end{aligned}
$$

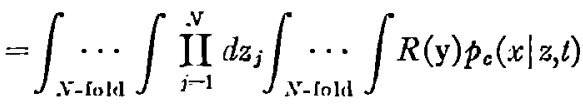

$$
\begin{aligned}
& \times p_{c}(\mathbf{z} \mid \mathbf{y}, \Delta l) \prod_{i=1}^{v} d y_{i} .
\end{aligned}
$$

Developing $R(\mathrm{y})$ in a Taýlor series in $\left(y_{i}-z_{i}\right)$,

$$
\begin{aligned}
& R(\mathbf{y})=R(\mathbf{z})+\sum_{i=1}^{y}\left(y_{i}-z_{i}\right) \frac{\partial R(\mathbf{y})}{\partial z_{i}} \\
&+\frac{1}{2} \sum_{i=1}^{N} \sum_{j=1}^{N}\left(y_{i}-z_{i}\right)\left(y_{j}-z_{j}\right) \\
& \\
& \times \frac{\partial^{2} R(\mathbf{y})}{\partial z_{j} \partial z_{j}}+0\left(|\mathbf{y}-\mathbf{z}|^{2}\right) .
\end{aligned}
$$

Substituting Eq. (1.14) into Eq. (1.13) and using Eq. (1.11), Eq. (1.1.3) becomes

$$
\begin{aligned}
& \frac{1}{\Delta t} \int_{N-\text { [o lal }} \ldots \int R(\mathrm{y})\left\{p_{c}(\mathrm{x} \mid \mathrm{y}, l+\Delta l)-p_{c}(\mathrm{x} \mid \mathrm{y}, l)\right\} \prod_{i-1}^{N} d y_{i} \\
& =\int_{X^{\prime}-\text { fold }} \ldots \int R(\mathbf{y})\left\{-\sum_{i-1}^{\mathbf{x}} \frac{\partial}{\partial y_{i}}\left[a_{,} p_{r}(\mathbf{x} \mid \mathbf{y}, t)\right]\right. \\
& \left.+\frac{1}{2} \sum_{i=1}^{N} \sum_{j=1}^{N} \frac{\partial^{2}}{\partial y_{i} \partial y_{j}}\left[b_{i j} p_{s}(\mathbf{x} \mid \mathbf{y}, l)\right]\right\} \prod_{i=1}^{N} d y_{i} .
\end{aligned}
$$


Taking the limit of the L.H. side as $\Delta t \rightarrow 0$, transposing the R.H. side, and substituting $\boldsymbol{p}_{c}$ for $p_{c}(\mathbf{x} \mid \mathbf{y}, l)$,

$$
\begin{aligned}
& \int_{x-\text { fold }} \ldots \int R(y)\left\{\frac{\partial p_{c}}{\partial l}+\sum_{i=1}^{N} \frac{\partial}{\partial y_{l}}\left[a_{i} p_{c}\right]\right. \\
& \left.-\frac{1}{2} \sum_{i=1}^{N} \sum_{j=1}^{x} \frac{\partial^{2}}{\partial y_{1}, \partial y_{j}}\left[b_{i j} p_{c}\right]\right\} \prod_{i=1}^{x} d y_{i}=0 .
\end{aligned}
$$

Since the function $R(\mathrm{y})$ is arbitrary, the quantity in the braces must vanish identically, giving

$$
\frac{\partial p_{c}}{\partial t}=-\sum_{i-1}^{x} \frac{\partial}{\partial y_{i}}\left[a_{i} p_{c}\right]+\frac{1}{2} \sum_{i=1}^{x} \sum_{j=1}^{Y} \frac{\partial^{\prime \prime}}{\partial y, \partial y_{j}}\left[b_{i j} p_{c}\right] .
$$

This parabolic, partial differential equation is known as the Fokker-Planck equation and is characteristic of diffusion processes of great importance to the fields of chemistry and thermodynamics.

The required solution of Eq. (1.17) is the positive solution satisfying the initial conditions

$$
p_{c}(\mathbf{x} \mid \mathbf{y}, l) \rightarrow \prod_{l=1}^{x} \delta\left(y_{i}-x_{i}\right) \quad\left(l \rightarrow 0^{\prime}\right),
$$

where $x_{i}$ is the initial value of $y_{1}$. 'Thus, the probability density approaches a delta function because the probability distribution (of $\mathbf{y}$ becoming $\mathbf{x}$ ) approaches unity when the time $t$ approaches zero (for $t \geqq 0$ ).

\section{Stationary Solution}

In certain problems, it may happen that with the passage of time the conditional probability $p_{c}(x \mid y, t)$ tends to a limiting stationary probability density $p(y)$. Simply stated, the probability density is no longer dependent on the time and the initial conditions. A solution to $p(y)$, if it exists, may be obtained from the Fokker-Planck equation by letting $t \rightarrow \infty$ and writing $\partial p / \partial t=0$. Thus, the equation for $p(y)$ is

$$
\begin{aligned}
\frac{1}{2} \sum_{i=1}^{x} \sum_{j=1}^{x} \frac{\partial^{2}}{\partial y_{2} \partial y_{j}}\left[b_{i j}(\mathrm{y}) p(\mathrm{y})\right] & \\
& -\sum_{i=1}^{x} \frac{\partial}{\partial y_{i}}\left[a_{i}(\mathrm{y}) p(\mathrm{y})\right]-0 .
\end{aligned}
$$

\section{APPLICATIONS}

\section{A. Single-Degree-of-Freedom System with Nonlinear Stiffness}

Consider the nonlinear oscillator whose response $x(l)$ is related to the excitation $f(t)$ by the following differential equation:

$$
\dot{x}+\beta \dot{x}+F(x)=f(t),
$$

where the excitation $f(l)$ is a stationary (Gaussian, white random process with a mean of zero; i.e.,

$$
\begin{aligned}
\langle f(l)\rangle & =0 ; \\
\left\langle f\left(l_{1}\right) f\left(l_{2}\right)\right\rangle & =\left(\mathrm{II}_{\mathbf{0}} / 2\right) \delta\left(t_{1}-t_{2}\right) .
\end{aligned}
$$

$\langle\cdots\rangle$ denotes an ensemble average, $W_{0}$ is the constant or white spectral density of the excitation, $\beta$ is the ratio of the linear, viscous damping coefficient to the mass, and $F(x)$ is the ratio of the nonlinear restoring force to the mass. If the nonlinear oscillator is attached to an immorable base, then $x(l)$ is the absolute displacement response of mass of the oscillator and $f(t)$ is the ratio of the exciting force (applied to the mass) to the mass. On the other hand, if the nonlinear oscillator is attached to an oscillating base, then $x(l)$ is the displacement response of the mass relative to the base and $f(t)$ is the negative of the vibratory acceleralion of the base.

When $f(t)$ is the ratio of the exciting force to the mass, $\mathrm{II}_{0}$ is the constant value of the mean square force (per unit mass) per unit bandwidth at frequency $\mathfrak{f}$ :

$$
I_{f f}(f)=\lim _{\substack{(\Delta f) \rightarrow 0 \\ l \rightarrow \infty}} \frac{\Delta \overline{\mathrm{j}(l)^{2}}}{(\Delta \bar{y})}
$$

where $(\Delta \mathfrak{i})$ is the bandwidth in cycles per unit time. When $f(l)$ is the negative of the vibratory acceleration of the base, then $\mathrm{I}_{0}$ is the constant value of $\mathrm{IH}_{f f}(\mathrm{i})$, the mean square acceleration per unit bandwidth.

I'riting $y_{1}=x$ and $y_{2}=\dot{x}$, Eq. (2.1) is equivalent to the following pair of first-order equations:

$$
\dot{y}_{1}=y_{2} ; \quad \dot{y}_{2}=-\beta y_{2}-F\left(y_{1}\right)+f(t) .
$$

The coefficients $a_{i}, b_{i j}$ in the Fokker-Planck equation may now be determined from $\mathrm{Eq}$. (1.11) once $A_{i}, B_{i j}$ of Eqs. (1.9) and (1.10) are determined. By knowledge of the physical system, $A_{1}=\left\langle\Delta y_{1}\right\rangle ; \quad A_{2}=\left\langle\Delta y_{2}\right\rangle ; B_{11}$ $=\left\langle\Delta y_{1}{ }^{2}\right\rangle ; \quad B_{12}=B_{21}=\left\langle\Delta y_{1} \Delta y_{2}\right\rangle ; B_{22}=\left\langle\Delta y_{2}{ }^{\prime}\right\rangle$. Making these substitutions into Eq. (1.11),

$$
\begin{aligned}
& a_{1}=\lim _{\Delta t \rightarrow 0} \frac{\left\langle\Delta y_{1}\right\rangle}{\Delta t}=y_{2}=\dot{x} ; \\
& a_{2}=\lim _{\Delta t \rightarrow 0} \frac{\left\langle\Delta y_{2}\right\rangle}{\Delta t} ; \\
& b_{11}=\lim _{\Delta t \rightarrow 0} \frac{\left\langle\Delta y_{1}^{2}\right\rangle}{\Delta t}=0 ; \\
& b_{12}=b_{21}=\lim _{\Delta t \rightarrow 0} \frac{\left\langle\Delta y_{1} \Delta y_{2}\right\rangle}{\Delta t}=0 ; \\
& b_{22}=\lim _{\Delta t \rightarrow 0} \frac{\left\langle\Delta y_{2}^{2}\right\rangle}{\Delta t} .
\end{aligned}
$$

Substituting Eq. (2.3) for $\left\langle\Delta y_{2}\right\rangle$ and $\left\langle\Delta y_{2}^{2}\right\rangle$ and utilizing 
$\tau$ as a clummy valiable in $t, a_{2}$ and $b_{22}$ become:

$$
a_{2}=\lim _{\Delta t \rightarrow t} \underbrace{\left\langle\left[y_{2}-F\left(y_{1}\right)\right] \Delta t+\int_{\ell}^{\ell+\Delta \ell} f(\tau) d \tau\right\rangle}_{-\beta}
$$

$$
\frac{\left\langle\left\{\left[-\beta y_{2}-F\left(y_{1}\right)\right] \Delta t+\int_{t}^{t+\Delta t} j(\tau) d \tau\right\}^{2}\right\rangle}{\Delta !}
$$

$$
\begin{aligned}
& =\lim _{\Delta \ell \rightarrow 0}\left\langle\left\{\left[-\beta y_{2}-F\left(y_{1}\right)\right]^{\prime \prime} \Delta t+2\left[-\beta y_{2}-F\left(y^{\prime}\right)\right]\right.\right. \\
& \left.\left.\times \int_{t}^{\ell+\Delta \ell} f(\tau) d \tau+\frac{1}{\Delta l} \iint f\left(\tau_{1}\right) j\left(\tau_{2}\right) d \tau_{1} d \tau_{2}\right\}\right) .
\end{aligned}
$$

Ipplying the conditions of Eq. (2.2),

$$
\begin{aligned}
& a_{2}=-\beta y_{2}-F\left(y_{1}\right)=-\beta \dot{x}-F(x) ; \\
& b_{22}=I^{-} 2 .
\end{aligned}
$$

In similar manner, it may be shown that higher moments are of $O(\Delta l)$ as $\Delta l \rightarrow 0$. Hence, the sistem satisfies the necessary conditions and is therefore governed by the Jokker -Planck equation.

Sulstituting Eq. (2.t) into Eq. (1.19) grives

$$
\frac{\|_{0}}{4} \frac{\partial^{3} p}{\partial y_{2}^{2}}-\frac{\partial}{\partial y_{1}}\left(y_{2} p\right)+\underset{\partial y_{2}}{\partial}\left\{\left[\beta y_{2}+F\left(y_{1}\right)\right]\right\} p=0
$$

This is the stationary form of Kramers equalion ${ }^{9}$ and has been solved independently by 'blenbeck, Caughey, ${ }^{10}$ Chuang and Kazada, "Ariaratnam, ${ }^{1.2}$ and Hu. ${ }^{\text {s }}$ We present the Caughey Wu solution here.

Equation (2.7) may be rewritten in the form

$$
\begin{array}{r}
{\left[\beta \frac{\partial}{\partial y_{2}}-\frac{\partial}{\partial y_{1}}\right]\left[y_{2} p+\frac{\amalg_{0}}{1 \beta} \frac{\partial p}{\partial y_{2}}\right]} \\
+\frac{\partial}{\partial y_{2}}\left[F\left(y_{1}\right) p+\frac{\amalg_{0}^{\prime}}{4 \beta} \frac{\partial p}{\partial y_{1}}\right]=0 .
\end{array}
$$

It is obvious that one solution of Eq. (2.8) will be

${ }^{9}$ H. A. Kramers, "Brownian Motion in a liekl of lorce and the Diflusion Morlel of Chemical Reactions," P'hysica 7, 284-304 $(1940)$.

10 \%. K. Caughey, "Response of Nonlinear Sy'stcms to Random Excitation," California Inst. 'Technol. Rept. 84 (1956) (unpublished); ibid., Rept. 90 (1957) (unjublished).

"K. Chuang and L. I". Kazada, "A Study of Non-Lincear Srstems with Randon Inputs," Trans. AIEE 78, Pairl II, 1100. 105 (1959).

12 S. T. Mriaratnam, "Random Vibration of Non-l.inear Su sprensions," J. Mech. Eng. Sci. 2, 3, 195-201 (1960).

${ }_{13}$ R. E. Oliver and T. Y. Wu, "Sled-Track Interaclion and : Rapid Methorl for Track-Alignment Measurement," Aeronaul ic al I:ngineering Rescarch Inc. Tech. Rept. 114, Part 2 (30 Junc 1958). obtained by requiring that $p\left(y_{1}, y_{2}\right)=p(x, \dot{x})$ satisfy the two equations

and

$$
y_{2} p+\frac{W_{0}}{4 \beta} \frac{\partial p}{\partial y_{2}}=0
$$

$$
F\left(y_{1}\right) p+\frac{\| r_{0}}{+\beta} \frac{\partial p}{\partial y_{1}}=0
$$

from which one readily obtains

$$
\begin{aligned}
p\left(y_{1}, y_{2}\right)=p(x, \dot{x}) & \\
& -\left(\exp \left\{-\frac{+\beta}{\mathrm{I}^{r}{ }_{0}}\left[\frac{y_{2}{ }^{2}}{2}+\int_{0}^{y_{1}} F(\zeta) d \zeta\right]\right\},\right.
\end{aligned}
$$

where $C$ is a normalizing constant and $\zeta$ is a dummy variable in $x$ or $y_{1}$. We see from this equation that the displacement and the velocity are statistical inrlependent ; i.c.,

$$
p\left(y_{1}, y_{2}\right)=p\left(y_{1}\right) \cdot p\left(y_{2}\right) .
$$

It is observed from Eq. (2.10) that $p\left(y_{1}, y_{2}\right)$ is (atussian in the velocity $y_{2}$. Indecd, it is identical with that for the linear problem $F\left(y_{1}\right)=\omega_{0}^{2} y_{1}$. Further, it is noted that the probability may be written $p\left(y_{1}, y_{2}\right)$ $=C \exp \left\{-4 \beta E W_{0},\right\}$, where $E$ is the total energy per unit mass of the systcm. This is simply the MaxwellBoltzmann distribution for an undamped antonomous oscillator whose mean kinctic energy per unit mass is $(T)=\Pi_{0}^{\circ}, \& \beta$.

An interesting consequence of Eq. (2.10) is that the system satisties the virial theorem for a rigid body moving under a conservative force ${ }^{14}$ :

$$
\bar{T}=\frac{1}{2} y_{1}^{\prime} \frac{\partial r^{\circ}}{\partial y_{1}},
$$

where the bar denotes a time average and $V$ is the potential energy per unit mass. If the process is ergodic, we may replace ensemble averages with time averages. Thus, $\bar{T}=\langle T\rangle=\left\langle\frac{1}{3} y_{2}{ }^{2}\right\rangle=\mathrm{IT}_{0}, 8 \beta$. Csing Eq. (2.10) in a like manner, $\overline{y_{1}}\left(\partial V \overline{\partial y_{1}}\right)=\left\langle y_{1} F\left(y_{1}\right)\right\rangle=I_{0}+\beta$. Thus, $\bar{T}=\overline{\frac{1}{2} y_{1}\left(\partial V, \partial y_{1}\right)}$, satisfying the virial theorem.

\section{Example}

To illustrate the application of Eq. (2.10), let us now show that the mean square displacement in a so-called "hardening spring" oscillator, whose characteristics are shown in Fig. 1, is always less than that of the corresponding linear oscillator. Let.

$$
F\left(y_{1}\right)=F(. x)=\omega_{1}:[x+\epsilon g(. x)],
$$

"IT. Golelstcin Classical Mechanics (Achlison-Mcsley ['ublish ing (o., Rearling, Mass., 1959), p. 70). 
where (a) $\epsilon>0$ and has dimensions of $x / g(x)$, (b) $g(x)=-g(-x)$, (c) $x g(x)>0$ for $|x|>0$, and $\omega_{0}$ is the undamped natural frequency of the corresponding linear system.

Let us evaluate the mean square displacement:

$$
\left\langle x^{2}\right\rangle=\int_{-\infty}^{+\infty} \int_{-\infty}^{+\infty} x^{2} p(x, \dot{x}) d x d \dot{x} .
$$

Substituting Eqs. (2.10) and (2.12) into Eq. (2.13) and integrating over $\dot{x}$,

where

$$
\left\langle x^{2}\right\rangle=\int_{-\infty}^{+\infty}\{x[x+\epsilon g(x)]-x \epsilon g(x)\} p(x) d x,
$$

$$
\begin{aligned}
p(x) & =C \exp \left\{-\frac{1}{\sigma_{x}^{2}} \int F(\zeta) d \zeta\right\} \\
& =C \exp \left\{-\frac{1}{\sigma_{x}^{2}}\left[\frac{x^{2}}{2}+\epsilon G(x)\right]\right\} \\
G(x) & =\int_{0}^{x} g(\zeta) d \zeta
\end{aligned}
$$

The mean square displacement and velocity of the corresponding linear system is $\sigma_{x}{ }^{2}=\Pi_{0} / 4 \beta \omega_{0}{ }^{2}$ and $\sigma_{\dot{x}}^{2}=W_{0} / 4 \beta$, respectively. [In Eq. (2.13), $x$ and $\dot{x}$ need not be statistically independent in order to integrate over $\dot{x}$.]

Integrating the first term of Eq. (2.14) by parts,

$$
\begin{aligned}
\left\langle x^{2}\right\rangle & =\sigma_{x}^{2}-\int_{-\infty}^{+\infty} x \epsilon g(x) p(x) d x \\
& =\sigma_{x}^{2}-\epsilon\langle x g(x)\rangle .
\end{aligned}
$$

Now, from (c) and (d) above,

$$
\left\langle x^{2}\right\rangle<\sigma_{x}^{2},
$$

where $\sigma_{x}^{2}$ is the mean square displacement for $\epsilon=0$. Hence, under conditions (a)-(c) above, the mean square displacement of a "hardening spring" nonlinear system is always less than that for the corresponding linear system.

\section{B. Extension to n-Degree-of-Freedom Systems}

Under certain restrictions, the foregoing theory may be extended to $n$-degree-of-freedom systems. Consider the system of equations

$$
\ddot{x}_{i}+\beta_{i} \dot{x}_{i}+\frac{1}{M_{i}} \frac{\partial \mho}{\partial x_{i}}=f_{i}(l) \quad(i=1,2, \cdots, n)
$$

Letting $y_{i}=x_{i}$ and $y_{i+n}=\dot{x}_{i}$, Eqs. (2.19) may be replaced by the $2 n$ system of equations:

$$
\dot{y}_{i}=y_{i+n}
$$

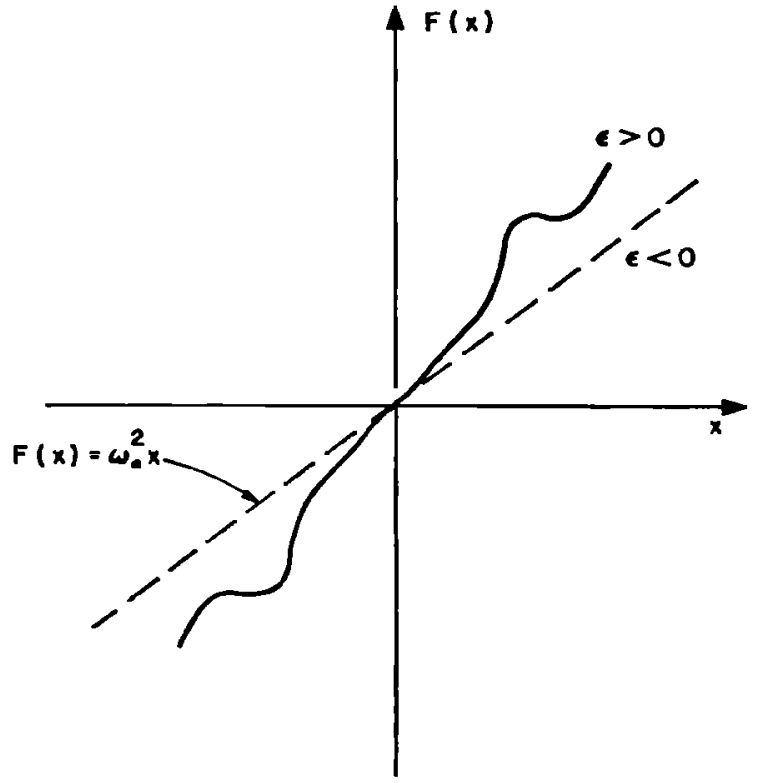

FIG. 1. Force vs displacement characteristic for a hardening spring oscillator $(\epsilon>0)$.

and

$$
\dot{y}_{i+n}=-\beta_{1} y_{i+n}-\frac{1}{M_{i}} \frac{\partial U}{\partial y_{i}}+f_{i}(l),
$$

where $V=V\left(y_{1}, y_{2}, \cdots, y_{n}\right)$ is the potential energy of the system, $f_{i}(l)$ are uncorrelated Gaussian, white random processes with means of zero; i.e.,

$$
\begin{aligned}
\left\langle f_{i}(l)\right\rangle & =0 ; \\
\left\langle f_{i}(t) f_{i}(l+\tau)\right\rangle & =W_{i i} \delta(\tau) / 2 ; \\
\left\langle f_{i}(t) f_{j}(l+\tau)\right\rangle & =0 \quad(i \neq j) .
\end{aligned}
$$

The coefficients $a_{i}, b_{i j}$ can be calculated using Eqs. (1.9)-(1.11), where $(i, j=1,2, \cdots, 2 n)$ :

$$
\begin{aligned}
a_{r} & =y_{i+n} ; \\
a_{i+n} & =-\beta_{i} y_{i+n}-\frac{1}{M_{i}} \frac{\partial U}{\partial y_{i}} ; \\
b_{i j} & =0 \quad(i \neq j) ; \\
b_{i+n, j+n} & =0 \quad(i \neq j) ; \\
b_{i i} & =0 \\
b_{i+n, i+n} & =W_{i i} / 2 .
\end{aligned}
$$

The Fokker-Planck equation for the stationary probability density function $p\left(y_{1}, y_{2}, \cdots y_{2 n}\right)$ is given by

$$
\begin{aligned}
& \sum_{i=1}^{n} \frac{W_{i i}}{4} \frac{\partial^{2} p}{\partial y_{i+n}{ }^{2}}-\sum_{i=1}^{n} \frac{\partial}{\partial y_{i}}\left(y_{i+n} p\right) \\
& \quad+\sum_{i=1}^{n} \frac{\partial}{\partial y_{i+n}}\left[\left(\beta_{i} y_{i+n}+\frac{1}{M_{i}} \frac{\partial v}{\partial y_{i}}\right) p\right]=0 .
\end{aligned}
$$


This equation may conveniently be rewritten in the Caughey-Wu form:

$$
\begin{aligned}
\sum_{i=1}^{n}\left[\left(\beta \frac{\partial}{\partial y_{i+n}}-\frac{\partial}{\partial y_{i}}\right)\left(y_{i+n} p+\frac{W_{i i}}{4 \beta_{i}} \frac{\partial p}{\partial y_{i+n}}\right)\right. \\
\left.+\frac{\partial}{\partial y_{i+n}}\left(\frac{1}{M_{2}} \frac{\partial U}{\partial y_{i}} p+\frac{W_{i i}}{4 \beta_{i}} \frac{\partial p}{\partial y_{i}}\right)\right]=0 .
\end{aligned}
$$

If we assume that $\Pi^{\prime}{ }_{11} 1 I_{i}, 4 \beta_{i}=K$, then

$$
\begin{aligned}
& p\left(y_{1}, y_{2} \cdots, y_{2 n}\right) \\
& =C \exp \left\{-\frac{1}{\left.\left.K^{\left[\frac{1}{2}\right.} \sum_{i=1}^{n} M_{i} y_{i+n^{2}}+v\right]\right\}}\right.
\end{aligned}
$$

As previously shown, Eq. (2.25) may also be written

$$
p\left(y_{1}, y_{2}, \cdots y_{2 n}\right)=C \exp \{-\mathcal{E} / K\},
$$

where $\mathcal{E}$ is the total energy stored in the system. It is noted from Eq. (2.24) that the probability is (iaussian in the velocities. The marginal probability is obtained by- integrating over the velocities and is given by

$$
p\left(y_{1}, y_{2}, \cdots y_{n}\right)=C^{\prime} \exp \{-v / K\} \text {. }
$$

It is interesting to note that as a consequence of Eq. (2.24) the system satisfies the general virial theorem ${ }^{14}$ :

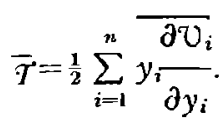

To prove this, we note that

$$
\begin{gathered}
\vec{T}=\langle T\rangle=\sum_{i=1}^{n} M_{i}\left\langle y_{i+n^{2}}\right\rangle=\frac{1}{2} n K ; \\
\sum_{i=1}^{n} \frac{\bar{\partial} U}{y_{i}}=\sum_{i=1}^{n}\left\langle y_{i} \frac{\partial U}{\partial y_{i}}\right\rangle=u K .
\end{gathered}
$$

'Thus, the system satisfies the virial theorem.

\section{Example}

To illustrate the use of Eq. (2.25), let us prove that the mean square displacements in a nonlinear $n$-degreeof-freedom system are smaller than those for the corresponding linear system when the nonlinearities are of the "hardening spring" type. Consider

$$
\ddot{x}_{i}+\beta \dot{x}_{i}+\omega_{i}^{2} x_{i}+\mu \frac{\partial I^{\top} \dagger}{\partial x_{i}}=f_{i}(l),
$$

where (a) $V^{\dagger}=V^{\dagger}\left(x_{1}, x_{2}, \cdots, x_{n}\right)$ is the potential energy per unit mass of the nonlinear terms; (b) $\mu>0$ and is dimensionless; (c) $x_{i}\left(\partial V^{\dagger} / \partial x_{i}\right)>0$; and (d) $f_{i}(l)$ are uncorrelated Gaussian, white random processes with the same spectral density $W_{0}$.
From this equation, the total potential energy per unit mass is

$$
V=\sum_{i=1}^{n} \frac{1}{2} \omega_{i}^{2} x_{i}^{2}+\mu V^{\prime}
$$

From Eq. (2.27),

$$
p\left(x_{1}, r_{2}, \cdots, r_{n}\right)=C^{\prime} \exp \left\{-t \beta V / \|_{u}\right\} .
$$

Now, the mean square displacement is

$$
\left\langle x_{j}^{2}\right\rangle=\int_{-\infty}^{+\infty} \cdots \int_{-\infty}^{+\infty} x_{j}^{2} p\left(x_{1}, x_{2,1}, \cdots, x_{n}\right) \prod_{i=1}^{n} d x_{i}
$$

Expressing

$$
x_{j}^{2}=\frac{x_{j}}{\omega_{j}{ }^{2}}\left(\omega_{j}^{2} x_{j}+\mu \frac{\partial I^{\prime}}{\partial x_{j}}-\mu \frac{\partial V}{\partial x_{j}}\right)
$$

and substituting this and Eq. (2.29) into Eq. (2.30),

$$
\left\langle x_{j}^{2}\right\rangle=\frac{1}{\omega_{j}^{2}}\left[\left\langle x_{j}\left(\omega_{j}^{2} \cdot,+\mu \frac{\partial V^{\dagger}}{\partial x_{j}}\right)\right\rangle-\left\langle\mu x_{j} \frac{\partial V^{\dagger}}{\partial x_{j}}\right\rangle\right] .
$$

Integrating the first term of Eq. (2.32) by parts and using Eq. (2.29),

$$
\left\langle x_{j}^{2}\right\rangle=W_{0} / 4 \beta \omega_{j}^{2}-\frac{\mu}{\omega_{j}^{2}}\left\langle x_{j} \frac{\partial V^{j}}{\partial x_{j}}\right\rangle .
$$

Using conditions (b) and (c) above, the second term is positive. Hence,

$$
\left\langle x_{j}{ }^{2}\right\rangle\left\langle\|^{2}{ }^{\prime} / \beta \omega_{j}{ }^{2}=\sigma_{x_{j}}{ }^{3},\right.
$$

just as is the case of the single-degree-of-freedom system of Eq. (2.18). Thus, under the above conditions, the mean square displacements of the svstem are smaller than those for the corresponding linear system.

\section{N-Degree-of-Freedom Quasilinear System}

Now consider the following system of equations in matrix notation:

$$
I\{\ddot{x}\}+\beta I\{\dot{x}\}+\left[1+2 \lambda V^{*}\right]\left[\Omega^{2}\right]\{x\}=\{f(l)\},
$$

where $\left[\Omega^{2}\right]$ is an $. * \times N$, symmetric, positive, definite matrix ; $V^{*}=\frac{1}{2}\{x\}^{T}\left[\Omega^{2}\right]\{x\}$ is the potential energy per unit mass of the linear system; $\lambda$ has dimensions of $1 / V^{*} ;\{f(t)\}$ is an $Y^{T}$-column vector of uncorrelated Gaussian, white random excitations with the same spectral density $W_{0}$.

Since $\left[\Omega^{2}\right]$ is symmetric, there exists an orthogonal transformation that reduces $\left[\Omega^{2}\right]$ to diagonal form. Let

$$
\{x\}=[\theta]\{\xi\}
$$

be this transformation, where $[\theta]^{T}[\theta]=I$. Thus, $[\theta]^{T}\left[\Omega^{2}\right][\theta]=\llbracket \omega^{2} \rrbracket$, a diagonal matrix. From this 
transformation,

$$
V^{*}=\frac{1}{2}\{\xi\}^{T}[\theta]^{T}\left[\Omega^{2}\right][\theta]\{\xi\}=\frac{1}{2}\{\xi\}^{T}\left[\left\{\omega^{2}\right]\{\xi\} .\right.
$$

Hence,

$$
V^{*}=\frac{1}{2} \sum_{k=1}^{N} \omega_{k}^{2} \xi_{k}^{2}
$$

Equation (2.35) may, therefore, be reduced to

$$
I\{\ddot{\xi}\}+\beta I\{\dot{\xi}\}+\left[1+\lambda \sum_{j=1}^{N} \omega_{j} \xi_{j}^{2}\right]\left[\omega^{2} \mathbb{W}\{\xi\}=\{Q(\ell)\},\right.
$$

where

$$
\{Q(l)\}=[\theta]^{T}\{f(l)\} .
$$

The correlation matrix is given by

$$
\begin{aligned}
\left\langle\left\{Q\left(l_{1}\right)\right\}\left\{Q\left(l_{2}\right)\right\}^{T}\right\rangle & =[\theta]^{T}\left\langle\left\{f\left(t_{1}\right)\right\}\left\{f\left(l_{2}\right)\right\}^{T}\right\rangle[\theta] \\
& =[\theta]^{T}\left(\frac{1}{2} W_{0}\right) \delta\left(l_{1}-t_{2}\right) I[\theta] \\
& =\frac{1}{2} W_{10} \delta\left(l_{1}-l_{2}\right) I .
\end{aligned}
$$

Thus, the $Q_{i}$ 's are uncorrelated.

The ith row of Eq. (2.39) is

$$
\ddot{\xi}_{i}+\beta \dot{\xi}_{i}+\omega_{2}^{2}\left[1+\lambda \sum_{j=1}^{n} \omega_{j}^{2} \xi_{j}^{2}\right] \xi_{i}=Q_{i}(l)
$$

This may be written as

$$
\ddot{\xi}_{i}+\beta \dot{\xi}_{i}+\partial V, \partial \xi_{i}=Q_{i}(i),
$$

where the total potential energy per unit mass is

$$
r=\frac{1}{2} \sum_{i=1}^{N} \omega_{l}^{2} \xi_{l}^{2}+-\sum_{4}^{\lambda} \sum_{l=1}^{N} \omega_{l=1}^{2} \omega_{m}^{2} \xi_{l}^{2} \xi_{m}{ }^{2} .
$$

Equation (2.39), therefore, satisties all the conditions laid down in Sec. $B$ on the $\boldsymbol{u}$-degree-of-freedom system. Hence,

$$
\begin{gathered}
p\left(\xi_{1}, \xi_{2}, \cdots \xi_{v}\right)=C^{\prime} \exp \left(-4,3 I / I_{0}\right\} . \\
\text { Example }
\end{gathered}
$$

Let us again prove that the mean square displacements are smaller than those for the corresponding linear system when the nonlinearities are of the "hardening spring" type. From Eq. (2.36),

$$
x_{i}=\sum_{j=1}^{N} \theta_{i}{ }^{j} \xi_{j}
$$

Therefore,

$$
\left\langle x_{i}{ }^{2}\right\rangle=\sum_{j=1}^{N} \sum_{k=1}^{N} \theta_{i}{ }^{i} \theta_{i}{ }^{k}\left\langle\xi, \xi_{k}\right\rangle
$$

Similar to Eqs. (2.13) and (2.30),

$$
\left\langle\xi_{j} \xi_{k}\right\rangle=\int_{x=\text { Iold }} \ldots \int \xi_{j} \xi_{k} p\left(\xi_{1}, \xi_{2}, \cdots, \xi_{N}\right) \prod_{i=1}^{x} d \xi_{i}
$$

But $p$ is symmetric in $\xi_{j}$ and $\xi_{k}$. Hence,

$$
\left\langle\xi_{j} \xi_{k}\right\rangle \equiv 0 \quad(j \neq k) .
$$

Therefore,

$$
\left\langle x_{i}^{2}\right\rangle=\sum_{j=1}^{V}\left(\theta_{i}\right)^{2}\left\langle\xi_{j}{ }^{2}\right\rangle
$$

where

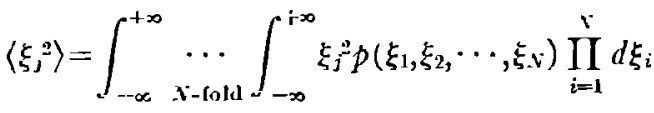

$$
\begin{aligned}
& =\int_{-\infty}^{+\infty} \cdots \int_{-\infty}^{+\infty} \frac{1}{\omega_{j}^{2}} \xi_{j}\left[\frac{\partial V}{\partial \xi_{j}}-2 \lambda \omega_{j}^{2} \xi_{j} V^{*}\right] \\
& \times p\left(\xi_{1}, \xi_{2}, \cdots, \xi_{N}\right) \prod_{i=1}^{N} d \xi_{i} .
\end{aligned}
$$

Integrating the first term of Eq. (2.51) by parts and utilizing Eq. (2.45),

$$
\left\langle\xi_{j}^{2}\right\rangle=\sigma_{\xi_{i}^{2}}{ }^{2}-\lambda\left\langle\xi_{j}{ }^{2}\left(\sum_{k=1}^{N} \omega_{k}^{2} \xi_{k}^{2}\right)\right\rangle .
$$

Since the second term of Eq. (2.52) is positive, then

$$
\left\langle\boldsymbol{x}_{i}{ }^{2}\right\rangle\left\langle\sum_{j=1}^{\mathrm{N}}\left(\theta_{i}^{j}\right)^{2} \sigma_{\xi_{j}}{ }^{2}=\sigma_{x_{j}}{ }^{2},\right.
$$

which proves the intended premise. An illustration of such a system is that of a massless string carrying $X$ identical particles of mass $M$. The problem was first solved by Caughey ${ }^{7}$ by using approximate techniques. Ariaratnam ${ }^{12}$ later solved the problem exactly by using essentially the technique outlined above.

\section{UNSOLVED PROBLEMS}

In this paper, a number of nonlinear problems have been discussed and solved. The impression should not be conveyed, however, that all problems relating to the response of nonlinear ststems with random excitation have been solved, for this is not the case. I should like to discuss a few problems that remain to be solved.

(a) Problems in which the nonlinearities involve velocity as well as displacement. For a number of years now, my students and I have worked diligently on this problem, with singular lack of success. It is apparent that the product-type solution as used in this paper is inadmissable for the case where the nonlinearity is velocity-dependent.

(b) Problems in mulidegree-of-freedom systems in which the exciling forces are correlaled and in which the spectral density is not related to the damping.

(c) Problems in which the exciting forces do not exhibit while spectra. In this case, it is not generally possible to solve the Fokker-Planck equation for the system by techniques implied in this paper. For linear systems, it has been possible to construct an equation similar to 
the Fokker-Planck equation governing the joint probability; but, at the present time, we have been unable to do this for any nonlinear system.

(d) Althongh w'e har'e succeeded in oblaining the stationary probability law for a class of nonlinear problems, a'e haie been generally unable to obtain the transitional probability laac. Without this transitional probability; it is generally impossible to obtain the correlation function and spectral density. Caughey and Dienes, ${ }^{15}$ however, have manayed to solve a rather trivial lirst-order problem in complete detail and obtain the spectral density. The techniques used in the solution of that problem do not appear to lend themselves to the solution of other nonlinear problems.

\section{APPENDIX A : UNIQUENESS OF A STATIONARY SOLUTION TO THE FOKKER PLANCK EQUATION}

(jiven the Fokker-Planck equation in the form

$$
\begin{aligned}
\frac{\partial p_{c}}{\partial t}+\frac{\partial}{\partial y_{k}}\left[a_{k}(y, z) p_{c}\right]+ & \frac{\partial}{\partial z_{k}}\left[c_{k}(y, z) p_{c}\right] \\
& -\frac{\partial^{2}}{2}\left[b_{k i}(y, z) p_{c}\right]=0,
\end{aligned}
$$

where $z_{i}=y_{i+n}, y_{k}$ are $m$ in number, $z_{k}$ are $n$ in number, and summation convention is implied. Lnder suitable assumptions, most of which were implied in the derivation of Eq. (1.1i), it is possible to show that there can be no more than one stationary solution. Integrals written as double integrals over $y$ and $z$ are used to designate the $(n+m)$-fold Lebesgue integrals over all the $z_{k}$ and $y_{k}$.

Though it is not shown here, it can be proved from the following assumptions that the order of integration is immaterial in all such integrals used herein.

\section{A. Restrictions}

Make the following requirements to Ey- (.11):

(a) $b_{k i}(y, s)$ represent the terms of a positive definite matrix.

(b) $\partial a_{k}(y, z)_{j} ; \partial y_{k}$ and $\partial c_{k}(y, z) / \partial z_{k}$ exist for every $y$ and $z$.

(c) $\partial^{2} b_{k_{i}}(y, z) / \partial z_{k} \partial z_{i}$ exists for every $y$ and $z$.

(d) The only solution to the problem given by the following equations is $x=$ constant :

$$
\begin{aligned}
& \text { (i) } a_{k}(y, z)\left[\partial_{x} \mathrm{l} / \partial y_{k}\right]=0 ; \\
& \text { (ii) } \partial x / \partial z_{k}=0 \quad(k=1,2, \cdots, n) ; \\
& \text { (iii) } x \geqq 0 .
\end{aligned}
$$

15 T. K. Caughey and J. K. Iienes, "Analysis of a Non-Linear First-()rder System with a White Noise Injul," J. Appll. Pliys. $32,2476-2479$ (1961).

\section{B. Further Restrictions}

We define a class of functions $\mathcal{U}$, such that $p_{c}$ is in $u$ if each of the following conditions are satisfied for all $t \geqq 0$.

(e) Each of the following terms is integrable (in the lebesgue sense) over all $y$ and $z$, and the multiple integrals may be evaluated by repeated integrations in any order. Here, no summation is implied:
(i) $p_{c}$;
(iv) $c \frac{\partial p_{c}}{\partial z_{k}}$;
(vii) $\frac{\partial b_{k_{t}}}{\partial z_{k}} \frac{\partial p_{c}}{\partial z_{i}}$;
(ii) $\frac{\partial p_{c}}{a_{k}-\frac{-}{\partial y_{k}}}$;
(v) $\frac{\partial c_{k}}{\partial z_{k}} p_{c}$;
(viii) $p_{c} \frac{\partial^{2} b_{k i}}{\partial z_{k} \partial z_{i}}$.
(iii) $\frac{\partial a_{k}}{\partial y_{k}} p_{c}$;
(vi) $b_{k i} \frac{\partial^{2} p_{c}}{\partial z_{k} \partial z_{i}}$

(f) Each of the following limits exists:
(i) $\lim _{y k \rightarrow 0} a_{k} p_{c}=0$;
(iii) $\lim _{2 k \rightarrow \infty} \frac{\partial p_{c}}{b_{k i}}=0$;
(ii) $\lim _{z k \rightarrow \infty} c_{k} p_{c}=0$;
(iv) $\lim _{i=k \rightarrow \infty} p_{c} \frac{\partial b_{k i}}{\partial z_{i}}=0$;

where no summation is implied and limits are taken with $t$ and all other $y$ and $z$ held fixed.

(g) $p_{c} \geqq 0$.

(h) At $I=0$, we have the relationship $\iint p_{c} d y d z=1$.

(i) $\partial p_{c} / \partial t$ is continuous in all variables.

\section{Proof of Uniqueness}

By utilizing all of the above restrictions, we may prove a number of theorems. The one that is important is given as follows:

Theorem 1: (iven that $p_{c 1}$ and $p_{c 1}$ each belong 10 $\mathcal{u}$, define $p_{c 3}, p_{c 4}$, and $x$ by

$$
\begin{aligned}
p_{c 3} & =a p_{c 1}+(1-a) p_{c 2} ; \\
p_{c 4} & =c p_{c 1}+(1-c) p_{c 2} \\
x & =p_{c 3} / p_{c 4}
\end{aligned}
$$

where we have $0<a<c<1$. Further, let us define

$$
X(t)=\iint x^{2} p_{c 4} d y d z
$$

Then, we have the relationship

$$
d X / d t=-2 \iint p_{c i} b_{k i} \frac{\partial x}{\partial z_{k}} \frac{\partial x}{\partial z_{i}} d y d z .
$$

l'roof: The proof of this theorem involves using 
restriction (e) to show that the integrals exist and restriction (f) to integrate by parts, after utilizing restrictions (e) and (i) to interchange differentiation and integration. By utilizing Theorem 1, it can be seen that, if both $p_{c 1}$ and $p_{c 2}$ are stationary solutions to the Fokker-Planck equation, then $X(t)$ must be constant. Hence, the integral of Eq. (A6) must be zero. Further, from the positive definite assumption on the $b_{k i}$ 's in restriction (a) and the fact that $p_{c 4}$ will be nonnegative, we see that the integral of Eq. (A6) cannot be zero unless the integrand is identically zero (using existence of the various derivatives to imply continuity). Hence, we arrive at the following lemma.

Lemma: If $p_{c 1}$ and $p_{c 2}$ each belong to $u$, with the added conditions that

and

$$
\partial p_{c 1} / \partial t=0
$$

$$
\partial p_{c 2} / \partial t=0,
$$

then we have the relationship that

$$
p_{c 4} b_{k i} \frac{\partial x}{\partial z_{k}} \frac{\partial x}{\partial z_{i}}=0 .
$$

From here, it is a simple matter to arrive at the uniqueness. Utilizing restrictions (a) and (d), we can see that $\partial x / \partial z_{k}=0$ for each $z_{k}$, and hence $x=$ constant. From the definition of $x$, we arrive at the fact that $p_{c 1}$ and $p_{c 2}$ must be related by a constant, which from restriction (h) must be unity.

Theorem 2: Given the added conditions that

and

$$
\partial p_{c 1} / \partial t=\partial p_{c 2} / \partial t=0
$$

we have

$$
p_{c 1}>0 \text {, }
$$

$$
p_{c 1}=p_{c 2} \text {. }
$$

Thus, if one stationary solution can be found that is nonzero everywhere, and if the above restrictions are satisfied (which they are in most problems of interest), then that solution is unique among the class of wellbehaved solutions.

\section{ACKNOWLEDGMENTS}

A. H. Gray, one of my students, is credited with the proof of the above Appendix. This work was supported in part by the Esso Educational Foundation. 